\title{
Remembered duration: Working memory and the reproduction of intervals
}

\author{
STEPHAN DUTKE \\ University of Kaiserslautern, Kaiserslautern, Germany
}

\begin{abstract}
On the basis of attention allocation models of time estimation, the role of working memory in prospective duration reproduction is explored. In four experiments, adult participants performed a counting task (duration, $400 \mathrm{sec}$ ) that allowed coordinative and sequential demands on working memory to be varied. After completing the counting task, the participants reproduced the time that they had worked on this task. It emerged that (1) increased coordinative demands on working memory (but not increased sequential demands) reduced the accuracy of prospective duration reproduction (Experiments 1 and 2), (2) presenting context information during the reproduction phase enhanced the accuracy of the reproduced duration (Experiment 3), and (3) individual differences in coordinative working memory capacity affected duration reproduction in the same direction as the experimental manipulation of coordinative task demands (Experiment 4). The results suggest that attention allocation models of time estimation may benefit from taking a more differentiated view of the types of attentional demands that affect temporal cognition.
\end{abstract}

The ability to process temporal information is central to the effective organization of behavior: "Time is the conscious experiential product of the processes that allow the (human) organism to adaptively organize itself, so that its behavior remains tuned to the sequential ... relations in its environment" (Michon, 1985, p. 20). Remembering the duration of past episodes is one of the main processes in this adaptation procedure. Information regarding the duration of intervals is essential for representing immediate processes of the environment (Zakay \& Block, 1997) and thus helps individuals to adapt their behavior to environmental processes. However, humans are extremely susceptible to temporal illusions, especially with regard to intervals lasting minutes or hours (see, e.g., Fraisse, 1984), and numerous experiments have been conducted to explore distortions in duration judgments (see Roeckelein, 2000, for a review).

The most successful hypothesis about the cognitive factors that influence duration judgments has focused on the allocation of attentional resources. As early as the 1920 s (Hülser, 1924), experimental evidence showed that directing attention to the "course of time" increased the subjective duration of temporal intervals, whereas distracting attention from time decreased subjective duration (see also Woodrow, 1933). In the 1970s, these phenomena were reanalyzed in terms of models in which a duality for the

The author thanks Wolfgang Schönpflug, Joachim Stöber, Ulrich von Hecker, and three anonymous reviewers for their helpful comments on earlier versions of the manuscript. Correspondence concerning this article should be addressed to S. Dutke, Department of Psychology, University of Kaiserslautern, Pfaffenbergstr. 95, D-67663 Kaiserslautern, Germany (e-mail: dutke@rhrk.uni-kl.de). processing of temporal and nontemporal information has been assumed. The assumption was that, as the cognitive capacity allocated to the processing of nontemporal information is increased, the remembered duration will either decrease or become unreliable (e.g., Hicks, Miller, Gaes, \& Bierman, 1977; Thomas \& Weaver, 1975; Zakay, 1989). Evidence confirming this hypothesis has been reported, for example, by Allen (1980), Brown and West (1990), Hawkes and Sherman (1972), Hicks and Brundige (1974), Hicks et al. (1977), Hicks, Miller, and Kinsbourne (1976), Kowal (1981), and Zakay (1989), using stimulus intervals ranging from 0.5 to $68 \mathrm{sec}$. Brown (1997) has provided a thorough review of studies showing that a variety of nontemporal tasks interfere with such temporal tasks as time estimation, time reproduction, and time production. It should be noted, however, that attention allocation theories are limited to explaining performance on prospective timing tasks - that is, tasks in which participants are informed in advance that they will have to estimate the duration of a specific interval (e.g., Zakay, 1993). When participants are given this instruction after the stimulus interval has already elapsed, time estimation is assumed to require different cognitive processes (e.g., Block, 1990).

A more recent version of the attention allocation explanation is the attentional gate model proposed by Zakay and Block (1997; Block \& Zakay, 1996). This model is based on the assumption that a mental pacemaker produces pulses at a rate that is affected by the organism's arousal. Whenever the organism directs attention to the course of time, a gate opens, allowing the stream of pulses to be transmitted to a cognitive counter. This counter can be controlled by cognitive processes, such as encoding strategies. The pulse count is transferred to working memory and to the so-called reference memory and is assumed 
to represent the duration of the interval to be estimated or remembered. The authors assume that attention directed to any nontemporal task will close the gate and, thus, reduce the number of pulses counted. Consequently, the internal temporal record contains gaps and is unreliable. Therefore, any increase in the difficulty of a concurrent nontemporal task filling the stimulus interval will distract resources from the operation of the gate or the counter, thus making it more difficult to reproduce the duration of this interval (Zakay \& Block, 1997, p. 15). Basically, this model is similar to the scalar timing model (Gibbon, Church, \& Meck, 1984) - in particular, with regard to the pacemaker component. However, the Block and Zakay (1996) model extends the scalar timing model by addressing also the effects of attention allocation and cognitive strategies.

Brown (1997) hypothesized that, if the attentional gate model is correct, interference between temporal and nontemporal processing should be bidirectional. Although he found that all nontemporal tasks administered in a series of experiments (mental arithmetic, visual search, and pursuit rotor tracking tasks) interrupted timing, the temporal task (concurrent time production) reduced performance in the mental arithmetic tasks only, and not in visual search or tracking. Brown interpreted this asymmetry with reference to Baddeley's (1986) working memory model. This model consists of three components: the so-called central executive and two dependent subsystems, the phonological loop for maintaining and manipulating verbal information and the visuospatial sketchpad for maintaining and manipulating visual images. ${ }^{1}$ The central executive bears some similarity to the supervisory attentional system (Norman \& Shallice, 1986) and is assumed to be responsible for controlling and coordinating the activities of the subsystems and for coordinating processes related to concurrent tasks (Baddeley, 1986, 1992). On the basis of the observation that all nontemporal tasks reduced performance on timing tasks, Brown concluded that timing generally suffers when the resources of the central executive are reduced by being directed to the coordinating of the temporal and the nontemporal tasks. On the other hand, the reduction of central executive resources entailed by dual-task coordination does not necessarily affect performance in the visual search or the tracking task, because both of these tasks are processed largely in one of the subsystems, meaning that the resources of the central executive are taxed to a lesser extent. Mental arithmetic was affected by timing, however, presumably because it taxed the phonological loop and the central executive (Brown, 1997; Verhaeghen, Kliegl, \& Mayr, 1997).

To summarize, according to Brown (1997), impaired temporal information processing can be conceptualized as the costs of dual-task coordination. It follows that increasing the difficulty of the nontemporal task should affect temporal processing only if it increases these coordination costs. This hypothesis differs from attention allocation models, which hold that any increase in the difficulty of the nontemporal task detracts processing resources from temporal information processing and, thus, impairs temporal information processing (Zakay \& Block, 1997, p. 15).

The problem with both positions, the unspecific (e.g., Zakay \& Block, 1997) and the specific (Brown, 1997) resource approaches, is that they are based on evidence from qualitatively different nontemporal tasks that probably differ in many more respects than just the coordination costs incurred. Thus, it is impossible to assess to what extent temporal processing is impaired because the coordination of the temporal and the nontemporal tasks reduces the central executive's resources (specific resource approach) or to what extent noncoordinative demands of the nontemporal task reduce the resources available for temporal information processing (unspecific resource approach).

To test these different predictions, in the present experiments, a concurrent nontemporal task was used (Dutke \& Stöber, 2001) that allowed coordinative and noncoordinative demands to be varied independently within the same task. The idea was that increasing the coordinative demands of the nontemporal task would also increase the complexity of between-task coordination. On the other hand, increasing the noncoordinative demands of the nontemporal task would not necessarily increase the complexity of between-task coordination. In the task used in the present experiments, the so-called counting task, lists of numbers were sequentially presented to the participant, who had been instructed to scan each list for a specific target number. The occurrences of this target number across the lists were counted according to specific instructions (see the Method section below). After finishing the counting task, the participants reproduced the time that they had spent working on that task. The coordinative demands of the counting task could be raised by increasing the number of independent targets to be counted at the same time. For example, the participant had to remember that the target number 16 had occurred twice and the target number 38 three times, while recognizing that the target number 67 was just occurring for the third time. In contrast to counting just one target number, this version of the task required intermediate processing results to be retained until further processing became necessary (Hagendorf \& Sá, 1995; Mayr \& Kliegl, 1993; Verhaeghen et al., 1997). The more the intermediate storage processes had to be coordinated with ongoing cognitive processes, the fewer the resources that could be allocated to the collecting of temporal pulses. The higher the coordinative demands of the concurrent counting task, the more unreliable time reproduction was likely to be. However, other aspects of the difficulty of the counting task could be manipulated independently of its coordinative demands - for example, its sequential demands on working memory (i.e., the number of processing steps that needed to be executed sequentially; Mayr \& Kliegl, 1993; Verhaeghen et al., 1997). The sequential demands of the counting task could be manipulated by varying the number of targets of the same type occurring in a set of lists. An increase in sequential demands meant that working memory contents had to be updated more frequently. However, increased sequential demands 
did not require additional resources to be allocated to the coordination of counting processes relating to different target numbers. Since an increase in sequential demands is not supposed to increase coordination costs, increased sequential demands were not expected to have any effects on duration reproduction. Nevertheless, both increased coordinative and sequential demands were expected to contribute to the difficulty of the counting task itself.

To summarize, according to Brown's (1997) specific resource view, coordinative, but not sequential, demands should affect prospective duration reproduction, because coordination taxes the resources required to complete the temporal record. In contrast, the attentional gate model (Block \& Zakay, 1996; Zakay \& Block, 1997) predicts that both types of demands will impair duration reproduction, because both require some attentional resources and, thus, contribute to the difficulty of a nontemporal task.

\section{EXPERIMENT 1}

The first experiment was designed to test the hypothesis that coordinative, but not sequential, demands on working memory impair participants' reproduction of the duration of a stimulus interval. Both coordinative and sequential demands were expected to increase the difficulty of the nontemporal task. The experiment consisted of two phases. First, the participants were presented with the counting task, which was used to manipulate coordinative and sequential demands independently. Before starting work on this task, they were informed that they would later be asked to reproduce its duration. Second, they reproduced the duration of the counting task by pressing a key when they felt that approximately the same amount of time had elapsed as they had spent on the task.

\section{Method}

\section{Participants}

Thirty-two female and 20 male students $(N=52)$ from different university departments were randomly assigned to four experimental conditions. Their ages ranged from 21 to 42 years $(M=28)$. All the students were paid for their participation $(€ 7)$. The data of 2 participants were excluded from the analyses because they misinterpreted the instructions for the counting task.

\section{Tasks and Design}

Counting task. The counting task-a continuous, computeradministered memory task (Dutke \& Stöber, 2001) — was administered as a nontemporal task. A list of 10 (randomly chosen) two-digit numbers was displayed for $8 \mathrm{sec}$ in the center of a computer monitor. After a pause of $2 \mathrm{sec}$, the next list of numbers was displayed. In total, 40 lists were presented. Thus, the total presentation time was $400 \mathrm{sec}$. The participants' task was to scan each list for (1) one target (low coordinative demands) or (2) one of three targets (high coordinative demands), to count the occurrences of each target, and to give a particular response when a target was displayed for the third time, as indicated below. The participants were instructed that the targets would appear randomly, with the restriction that there would not be more than one target per list.

Coordinative demands. In the low coordinative demands condition, the participants were asked to count the occurrences of a single target number (the number 16) across the lists. After inspecting each list, the participants responded to the question of whether or not the number 16 had occurred for the third time. If a list did not contain the target or the target appeared for the first or second time, they were to press a key marked "no." When the target occurred for the third time, they were to press a key marked "yes" and to start counting from zero again (so that the next occurrence of the target would again be counted as its first appearance). Then the "yes" key had to be pressed for a second time for the presentation of the lists to be continued. There was no time limitation between pressing the "yes" key for the first and the second time. In this (low coordinative demands) condition, (1) refreshing and (2) updating the tally and (3) responding could be executed sequentially, with a minimum of intermediate storage requirements. In the high coordinative demands condition, however, the participants had to manage three tallies, one for each target (the numbers 16, 38, and 67). When the participants detected a target, the tally for this (and only this) target was increased by one. After inspecting each list, the participants responded to the question of whether or not one of the numbers 16 , 38 , or 67 had occurred for the third time. If a list did not contain a target or if a target appeared for only the first or second time, the participants were to press a key marked "no." When a target appeared for the third time, the participants were to press a key marked "yes," specify which target had appeared for the third time by pressing the respective key (i.e., a key marked " 16 ," “38," or “67”), and start counting this target from zero again. The tallies for the other two targets remained unchanged. There was no time limitation for specifying the target. In this high coordinative demands condition, the working memory system had to coordinate storing, refreshing, updating, and responding - without confusing the tallies for the three target numbers.

Sequential demands. The counting task allowed for sequential demands to be varied independently of coordinative demands by manipulating the frequency with which targets appeared. In the present experiment, two sequential demands conditions were implemented. In the low sequential demands condition, 14 out of 40 lists $(35 \%)$ contained a target. In the high sequential demands condition, 27 out of 40 lists $(68 \%)$ contained a target. High sequential demands meant that the working memory system had to deal with a higher rate of updating the results of the counting process.

Duration reproduction task. After the counting task, the participants had to press a key labeled "s" to start the duration reproduction task, wait as long as they felt they had spent on the counting task, and finally press a key labeled "e" ("end") when they felt that the same amount of time had elapsed. The participants were instructed prospectively - that is, they were informed about the duration reproduction task before starting the counting task. With regard to the counting task, the participants were instructed to count the targets as accurately as possible, while bearing in mind that they would be expected to reproduce the duration of the counting task as closely as possible after completing it.

Design. The two levels of coordinative demands (one vs. three target numbers) were combined with the two levels of sequential demands (a total of 14 vs. 27 occurrences), resulting in a $2 \times 2$ between-subjects design.

\section{Procedure}

The experiment was run in individual sessions. The participant was asked to leave his or her watch outside the experiment room and was informed that the experiment consisted of two parts: the counting task and the duration reproduction task. For both parts, the instructions were presented orally by the experimenter, accompanied by a demonstration on the computer screen. The experimenter left the room after ensuring that the participant had understood both tasks, and the participant commenced the counting task. Once the counting task had been completed, an instruction was displayed on the screen informing the participant to start the duration reproduction task without delay. After the duration reproduction task, the experimenter came back into the room and interviewed the participant. 


\section{Dependent Variables}

The reproduced duration (measured in seconds) was diminished by the objective duration of the stimulus interval. The duration reproduction error was assessed by dividing this difference by the objective duration of the stimulus interval. Thus, negative values indicated a relative underestimation, positive values a relative overestimation. After reproducing the stimulus interval, the participant gave a verbal estimation of the number of lists presented during the stimulus interval.

\section{Results}

Almost all the participants underestimated the duration of the counting task. As was predicted, the duration reproduction error was larger under high coordinative demands than under low coordinative demands (Table 1) $\left[F(1,46)=7.91, M S_{\mathrm{e}}=0.032, p<.01 ; \operatorname{partial} \eta^{2}=.15\right]$, the reproduced duration being shorter under high than under low coordinative demands. However, the duration reproduction error was unaffected by the manipulation of sequential demands $[F(1,46)=0.69]$. The estimated number of lists presented during the stimulus interval was positively correlated with the duration reproduction error $(r=.45, p<.01)$. The more lists the participants believed had been presented during the counting task, the longer the reproduced duration (and the "less negative" the duration reproduction error).

In the counting task, more errors were committed under high coordinative demands than under low coordinative demands [Table 1; $F(1,46)=17.62, M S_{\mathrm{e}}=9.93, p<$ $.001] .{ }^{2}$ Thus, coordinative demands affected counting performance in the same direction as they did performance in the duration reproduction task. Sequential demands also affected the number of counting errors $[F(1,46)=$ $\left.9.58, M S_{\mathrm{e}}=9.93, p<.01\right]$. The participants committed more counting errors under high sequential demands than under low sequential demands (Table 1). The significant interaction $\left[F(1,46)=7.38, M S_{\mathrm{e}}=9.93, p<.01\right]$ reveals that the highest number of counting errors occurred under high coordinative and high sequential demands, whereas the lowest number of errors was observed in the condition with low coordinative and low sequential demands.

\section{Discussion}

Both increased coordinative and increased sequential demands on working memory intensified the difficulty of the counting task, as demonstrated by higher numbers of counting errors under both types of demands. Thus, increasing demands on each of these dimensions can be assumed to absorb processing resources. However, increased coordinative demands affected the accuracy of duration reproduction, but increased sequential demands did not. Increasing the frequency with which the contents of working memory were to be updated did not impair later duration reproduction, whereas coordinating the maintenance of different pieces of information did. This finding qualifies the assumption of the attentional gate model that any nontemporal task demands will detract cognitive capacity from the time estimation task and corroborates Brown's (1997) specific resource account.
Table 1

Mean Duration Reproduction Error and Mean Counting Error Index (With Standard Deviations) in Experiments 1 and 2

\begin{tabular}{|c|c|c|c|c|c|}
\hline \multirow{2}{*}{$\begin{array}{c}\text { Coordinative } \\
\text { Demands }\end{array}$} & \multirow{2}{*}{$\begin{array}{c}\text { Sequential } \\
\text { Demands }\end{array}$} & \multicolumn{2}{|c|}{$\begin{array}{c}\text { Duration } \\
\text { Reproduction } \\
\text { Error }\end{array}$} & \multicolumn{2}{|c|}{$\begin{array}{l}\text { Counting } \\
\text { Error }\end{array}$} \\
\hline & & $M$ & $S D$ & $M$ & $S D$ \\
\hline \multicolumn{6}{|c|}{ Experiment 1} \\
\hline \multirow[t]{2}{*}{ Low } & Low & -.36 & .16 & 0.86 & 0.98 \\
\hline & High & -.45 & .19 & 1.19 & 1.22 \\
\hline \multirow{2}{*}{ High } & Low & -.55 & .11 & 2.18 & 2.29 \\
\hline & High & -.54 & .22 & 7.37 & 5.59 \\
\hline \multicolumn{6}{|c|}{ Experiment 2} \\
\hline \multirow[t]{2}{*}{ Low } & Low & -.26 & .18 & 1.73 & 2.07 \\
\hline & High & -.26 & .21 & 0.98 & 0.98 \\
\hline \multirow[t]{2}{*}{ High } & Low & -.46 & .24 & 7.35 & 4.24 \\
\hline & High & -.51 & .21 & 12.74 & 7.94 \\
\hline
\end{tabular}

A significant correlation was found between the number of lists the participants believed that they had seen during the stimulus interval and the reproduced duration. The estimated frequency of lists may be interpreted as a verbal temporal cue (Woodrow, 1951) constructed from the internal representation of the experienced duration. A correlation between such a temporal cue and time reproduction is consistent with both the specific and the unspecific resource approaches, since both positions hold that a reduction in resources impairs the functioning of the so-called gate and, thus, reduces the pulse count. Since attention allocation models postulate that this pulse count is the main informational basis for estimating an interval's duration, both the duration reproduced and the verbal cue of the experienced duration must be rooted in the number of pulses counted. If the duration reproduced and the estimated number of lists are derived from the same informational basis, they should indeed be correlated.

Many studies have shown that the cognitive strategies used in time judgment tasks (Guay \& Wilberg, 1983) are influenced by strategies such as internal counting (Wilkening, Levin, \& Druyan, 1987). In the present study, however, it is extremely unlikely that the participants kept a precise tally of the number of lists, because this would have interfered with their task of target counting. In postexperimental interviews, all the participants reported that it was impossible for them to keep count of the stimulus lists. Thus, it is unlikely that the duration reproduced was based on the participants' tally of the number of lists presented.

Although presentation times and interstimulus intervals did not differ between the conditions, the experimental procedure allowed the total duration of the stimulus interval to vary. After a yes response (meaning that a target was judged to have occurred for the third time), the program controlling the experimental procedure either started a short dialogue in which the participant identified the respective target (high coordinative demands condition) or required an additional key to be pressed, upon which the presentation of lists was continued (low coordinative demands condition). The sum of these interrup- 
tion times was greater under high coordinative demands $(M=82.0 \mathrm{sec}, S D=39.6)$ than under low coordinative demands $(M=30.8 \mathrm{sec}, S D=12.4)$, although the number of stimulus lists requiring a yes response was equal in both conditions. It is reasonable to assume that these pauses were used by the participants to recapitulate the counting process and to update the current tally before returning to the lists. This may have attenuated the impact of the coordinative demands manipulation. Moreover, it led to an unintended confounding of the duration of the stimulus interval and the coordinative demands factor, because the longer interruptions in the high coordinative demands condition contributed to the total duration of the stimulus interval.

\section{EXPERIMENT 2}

The second experiment was designed to replicate the results of the first experiment, utilizing a different response procedure in the counting task in order to avoid the confounding of coordinative demands and the duration of the stimulus interval. The new response procedure guaranteed that the duration of the stimulus interval did not differ between the experimental conditions and that the participants had no opportunity to evade the time limitations in the presentation of the lists. It was hypothesized that, under this condition, it would be harder for the participants to compensate for high coordinative demands. Therefore, the impact of high coordinative demands on the duration reproduction error was expected to be stronger than in the first experiment.

\section{Method}

\section{Participants}

Twenty female and 36 male students $(N=56)$ from different university departments were randomly assigned to four experimental conditions. Their ages ranged from 16 to 34 years $(M=25)$. All the students were paid for their participation $(€ 7)$.

\section{Design and Procedure}

The study design, procedure, and dependent variables were the same as those in the first experiment, with one exception: Instead of the two-stage response procedure after yes responses (first experiment), a single-stage response procedure was introduced. Under low coordinative demands, each time a stimulus list was presented, the participants had to decide whether or not they had seen the target number for the third time, responding by pressing keys labeled "yes" and "no." Under high coordinative demands, the participants had to decide between four alternatives (no, yes [16], yes [38], and yes [67]), responding by pressing one of four keys labeled "no," "16," "38," and "67." Accordingly, the duration of the stimulus interval was constant at $400 \mathrm{sec}$.

\section{Results}

As in the first experiment, most of the participants underestimated the duration of the stimulus interval. The duration reproduction error was larger $[F(1,52)=15.72$, $M S_{\mathrm{e}}=0.045, p<.001$; partial $\eta^{2}=.23$ ] under high coordinative demands than under low coordinative demands (Table 1). However, the duration reproduction error was unaffected by the manipulation of sequential demands
$[F(1,52)=0.25]$. The estimated number of lists presented during the stimulus interval was positively correlated with the duration reproduction error $(r=.36, p<.01)$. As in Experiment 1, the reproduced duration was longer (and the duration reproduction error "less negative"), the more lists the participants believed had been presented during the counting task.

In the counting task, more errors were committed under high coordinative demands than under low coordinative demands [Table $1 ; F(1,52)=48.99, M S_{\mathrm{e}}=21.56, p<$ $.001]$. Thus, coordinative demands affected counting performance in the same direction as they did performance in the duration reproduction task. Sequential demands also affected the number of counting errors. Whereas the main effect of sequential demands did not reach the conventional level of significance $[F(1,52)=3.05, p=$ $.067]$, the interaction term was significant $[F(1,52)=$ $\left.6.11, M S_{\mathrm{e}}=21.56, p<.05\right]$. The participants in the high coordinative demands condition committed more counting errors under high sequential demands than under low sequential demands $[t(26)=-2.24, p<.05]$.

In postexperimental interviews, all the participants reported that it was impossible for them to keep count of the stimulus lists while counting the targets.

\section{Discussion}

The results of Experiment 1 were replicated. Increased coordinative demands on working memory decreased the reproduced duration and increased the duration reproduction error. Again, manipulating sequential demands did not affect the accuracy of the reproduced duration.

The impact of high coordinative demands was slightly stronger under the new response procedure ( $\Delta$ partial $\left.\eta^{2}=.08\right)$. This might be because the participants in Experiment 2 were forced to keep up with the set rhythm of stimulus presentation, without the opportunity to recover in self-regulated pauses.

In Experiments 1 and 2, the errors committed in the counting task indicated that both types of working memory demands - complexity of coordination and frequency of working memory updates - increased the difficulty of the counting task. However, only coordinative demands affected the accuracy of duration reproduction. This again corroborates Brown's (1997) hypothesis that it is primarily coordination costs that affect temporal information processing.

As in Experiment 1, the estimated number of lists presented during the stimulus interval correlated with the reproduced duration. This corroborates the interpretation that the reproduction of time and of verbal temporal cues may be rooted in the same informational basis, as is postulated by the specific and the unspecific resource approaches.

\section{EXPERIMENT 3}

Experiments 1 and 2 showed that increased coordinative demands on working memory during the stimulus interval reduce the subjective duration of this interval. Both the attentional gate model (Block \& Zakay, 1996; Zakay 
\& Block, 1997) and Brown's (1997) modification hold that reduced subjective duration results from a fragmentary internal record of temporal pulses. It is assumed that insufficient mental resources either impair the operation of the so-called gate, meaning that the stream of temporal pulses is interrupted too often, or impair the operation of the so-called cognitive counter, meaning that an unreliable count of temporal pulses is transferred to the reference memory. This proposition can be investigated by trying to "repair" the deficient informational basis upon which duration reproduction draws after the stimulus interval. With regard to long stimulus intervals (lasting several seconds or even minutes), at least, it is assumed that the accuracy of duration reproduction depends not only on the encoding conditions during the stimulus interval, but also on the conditions under which the duration is reproduced (Hemmes, Brown, \& Kladopoulos, 2004). In the present experiment, the duration of the stimulus interval was reproduced under two different conditions. In one condition, the reproduction was carried out solely on the basis of the internal temporal record, as in the first two experiments. In the other condition, the reproduction process was supported by temporal context cues that partially reinstantiated the encoding situation. The availability of temporal context cues during reproduction was expected to compensate for the deficiencies in the internal temporal record caused by high coordinative demands during the stimulus interval.

\section{Method}

\section{Participants}

Fifty women and 30 men $(N=80)$, students of psychology in their first term at the university, were randomly assigned to four experimental conditions. Their ages ranged from 18 to 43 years $(M=$ $25)$. All the students were paid for their participation $(€ 7)$.

\section{Design and Procedure}

High and low coordinative demands of the counting task were operationalized as in the second experiment. In both conditions, a total of 18 targets occurred.

There were two conditions in the duration reproduction task, one being identical with the procedure in the previous experiments. Here, the participant reproduced the duration of the stimulus interval, relying solely on imagery, without any external support. In the second condition, the context information condition, the participants were asked to reproduce the stimulus interval's duration within a context similar to that of the stimulus interval. The participants, who were seated in front of the computer monitor, were shown stimulus lists similar to those presented during the stimulus interval but were instructed not to count any targets. In fact, these lists did not even contain the previous target numbers. In contrast to the stimulus interval, the lists did not disappear after a fixed interval, but it was left to the participants to call up each new list by pressing the space bar. The participants were instructed to do this in the same "rhythm" as in the stimulus interval, for the estimated length of the stimulus interval. It was expected that the presentation of a context that allows aspects of the stimulus presentation to be recreated and actions similar to those of the stimulus interval to be executed would facilitate accurate temporal reconstruction. For example, we can expect the presentation time for a stimulus list to be reconstructed more accurately when the participant is shown a real stimulus list and tries to reenact the actions executed during the stimulus interval, such as scanning the list, pressing the correct key, waiting for the next list, and so on. Irrespective of the initial quality of the duration representation, the perception of the stimulus material and the actual execution of the associated actions are assumed to result in a more accurate reconstruction of the stimulus interval, as compared with a reproduction without any external support.

The two levels of both independent variables were combined in a 2 (high vs. low coordinative demands) $\times 2$ (with vs. without context information) between-subjects design. The procedure was the same as that in the second experiment, except for the manipulation of the duration reproduction procedure.

\section{Results}

Replicating the results of the first two experiments, the duration reproduction error was larger under high coordinative demands than under low coordinative demands [Table 2; $\left.F(1,76)=12.57, M S_{\mathrm{e}}=0.051, p<.01\right]$. The context information factor also affected the duration reproduction in the expected direction. Duration reproduction was more accurate with context information than without [Table 2; $F(1,76)=12.28, M S_{\mathrm{e}}=0.051, p<$ $.01]$. The two factors did not interact $[F(1,76)=0.23]$. As in the previous experiments, the duration reproduction error was positively correlated with the estimated number of stimulus lists $(r=.44, p<.001)$. The correlation between the duration reproduction error and the number of lists the participants actually requested to be displayed during the reproduction phase was even higher $(r=.61$, $p<.001)$.

As was expected, counting errors occurred more frequently under high coordinative demands than under low coordinative demands [Table $2 ; F(1,76)=37.04, M S_{\mathrm{e}}=$ $13.93, p<.001]$. Thus, coordinative demands affected counting performance in the same direction as performance in the duration reproduction task. Moreover, the number of counting errors did not differ between the group that received context information during reproduction and the group that did not.

In postexperimental interviews, all the participants reported that it was impossible for them to keep count of the stimulus lists while counting the targets.

\section{Discussion}

Again, the detrimental effects of high coordinative demands on performance in the counting task and on the accuracy of duration reproduction were replicated. However, it was also demonstrated that the accuracy of duration reproduction was not exclusively a function of the encoding

Table 2

Mean Duration Reproduction Error and Mean Counting Error Index (With Standard Deviations) in Experiment 3

\begin{tabular}{|c|c|c|c|c|c|}
\hline \multirow{2}{*}{$\begin{array}{c}\text { Coordinative } \\
\text { Demands }\end{array}$} & \multirow{2}{*}{$\begin{array}{c}\text { Context } \\
\text { Information }\end{array}$} & \multicolumn{2}{|c|}{$\begin{array}{c}\text { Duration } \\
\text { Reproduction } \\
\text { Error } \\
\end{array}$} & \multicolumn{2}{|c|}{$\begin{array}{c}\text { Counting } \\
\text { Error }\end{array}$} \\
\hline & & $M$ & $S D$ & $M$ & $S D$ \\
\hline \multirow[t]{2}{*}{ Low } & Without & -.27 & .23 & 0.63 & 1.39 \\
\hline & With & -.07 & .23 & 1.20 & 1.90 \\
\hline \multirow[t]{2}{*}{ High } & Without & -.42 & .25 & 6.90 & 6.60 \\
\hline & With & -.27 & .18 & 5.08 & 2.58 \\
\hline
\end{tabular}


activity but was also influenced by the support provided for the reconstruction process itself (for a related result, see Hemmes et al., 2004). The two influences seemed to be independent of each other, since there was no significant interaction between coordinative demands and the context information factor. This pattern is consistent with the assumption that the reproduction of time intervals is based on the availability of an internal temporal record, the accuracy of which may vary. If the record is incomplete, the time reproduction error increases. However, the provision of temporal context cues during reproduction may compensate for the incompleteness resulting from high coordinative demands during the stimulus interval.

This interpretation also corresponds to the notion that duration reproduction (of long intervals, at least) may be based on a dynamic mental model of the stimulus interval (Freyd, 1992; Michon, 1990). This temporal mental model allows what was experienced during the stimulus interval to be mentally simulated and, hence, its duration to be reproduced. When intervals last a number of minutes, this simulation may take the form of mentally replaying the actions or imaging the events that occurred during the stimulus interval (Poynter, 1989; see also Decety, Jeannerod, \& Prablanc, 1989; Munzert, 2002). This interpretation was further corroborated by the observation that the number of lists requested during duration reproduction was substantially correlated with the reproduced duration.

\section{EXPERIMENT 4}

The previous experiments demonstrated how experimentally induced coordinative demands on the working memory system affect the accuracy of duration reproduction. This manipulation can be understood as an artificial resource limitation imposed on the central executive of the working memory system. The higher the coordinative demands of the counting task, the fewer the resources that can be allocated to preparing for the duration reproduction task. However, substantial interindividual differences in working memory capacity also explain a great deal of the individual variance in cognitive performance in reasoning or intelligence tests, for example (cf. Kyllonen \& Christal, 1990; Süß, Oberauer, Wittmann, Wilhelm, \& Schulze, 2002). Therefore, it is hypothesized that the accuracy of duration reproduction covaries with naturally occurring individual differences in coordinative working memory capacity, in the same way as it does with experimentally induced limitations of this resource.

\section{Method}

\section{Participants}

Thirty-one women and 13 men $(N=44)$, students from different university departments, volunteered for this experiment. Their ages ranged from 19 to 48 years $(M=26)$. All the students were paid for their participation $(€ 7)$.

\section{Tasks and Design}

The participants were tested for their coordinative working memory capacity, using the verbal coordination task (Oberauer, 1993).
This task was designed to measure the ability to simultaneously access several distinct elements and to coordinate them into a new, coherent mental structure. In a comprehensive study, Oberauer, Süß, Schulze, Wilhelm, and Wittmann (2000) examined the reliability and validity of this and other tasks designed to capture various functional facets of working memory. For the verbal coordination task, they reported a Cronbach's alpha of .84, as well as strong correlations with various working memory marker tasks and with the reasoning ability component in intelligence test batteries.

In the verbal coordination task, participants are presented with a row of boxes at the center of a computer screen. A letter is displayed in one of these boxes for $1,150 \mathrm{msec}$. Another letter is then displayed in another of the boxes. This process is repeated until a letter has been shown in each of the boxes. The participants are instructed (1) to decide whether or not the complete set of letters represents a German word (read from left to right or from right to left) and (2) to write down the letters on a response sheet showing the same number of boxes as displayed on the screen. Since only one letter is visible at a time, an internal representation of the complete stimulus array has to be constructed for the lexical decision to be made. This task demands storage and coordination resources to be mobilized simultaneously, because the participant has to (1) memorize the letters presented so far, (2) identify the stimulus letter currently presented, (3) integrate this perception into an overall representation of the stimuli already held in the memory, and finally (4) make the lexical decision. To ensure that the lexical decision is made on the basis of this internal representation and not on the basis of letters noted down on the response sheet, the participants have to choose between two lists provided on the sheet, one for words and one for nonwords, before the response can be made. Fifteen items are presented, with four to eight letters each. The percentage of letters reproduced in the correct configuration, out of the total number of letters (90) across all items, is used as the performance score.

The median performance score in the verbal coordination task was used to split the sample into a subgroup with higher coordinative working memory capacity $(M=79.1, S D=5.7)$ and a subgroup with lower working memory capacity $(M=56.5, S D=$ 10.1). Half of the participants in each group were randomly selected to work on the one-target version of the counting task (low coordinative demands); the other half worked on the three-target version (high coordinative demands).

\section{Procedure}

In a first session, the participants were tested with the verbal coordination task. After being assigned to the experimental conditions, the participants were invited to a second session, in which they performed the counting task and the duration reproduction task. The rest of the experimental procedure was the same as that in Experiment 2.

\section{Results}

The coordinative demands had the expected main effect on the duration reproduction error $[F(1,40)=5.47$, $\left.M S_{\mathrm{e}}=0.033, p<.05\right]$. Duration reproduction was more accurate under low coordinative demands than under high coordinative demands (Table 3 ). The main effect of working memory capacity was also significant $[F(1,40)=$ $\left.3.93, M S_{\mathrm{e}}=0.033, p<.05\right]$. The participants with high coordinative working memory capacity reproduced the duration of the counting task more accurately than did the participants with lower coordinative capacity (Table 3). The insignificant interaction term suggests that the two effects were independent of each other $[F(1,40)=0.57]$.

Parallel effects were found for the counting error index. More errors occurred under high coordinative demands 
Table 3

Mean Duration Reproduction Error and Mean Counting Error Index (With Standard Deviations) in Experiment 4

\begin{tabular}{|c|c|c|c|c|c|}
\hline \multirow{2}{*}{$\begin{array}{c}\text { Coordinative } \\
\text { Demands }\end{array}$} & \multirow{2}{*}{$\begin{array}{l}\text { Coordinative } \\
\text { Working } \\
\text { Memory } \\
\text { Capacity }\end{array}$} & \multicolumn{2}{|c|}{$\begin{array}{c}\text { Duration } \\
\text { Reproduction } \\
\text { Error } \\
\end{array}$} & \multicolumn{2}{|c|}{$\begin{array}{l}\text { Counting } \\
\text { Error }\end{array}$} \\
\hline & & $M$ & $S D$ & $M$ & $S D$ \\
\hline \multirow[t]{2}{*}{ Low } & Low & -.35 & .20 & 0.82 & 0.75 \\
\hline & High & -.20 & .23 & 0.36 & 0.50 \\
\hline \multirow[t]{2}{*}{ High } & Low & -.44 & .14 & 4.00 & 2.61 \\
\hline & High & -.37 & .13 & 2.82 & 1.33 \\
\hline
\end{tabular}

than under low coordinative demands (Table 3$)[F(1,40)=$ $\left.37.25, M S_{\mathrm{e}}=2.35, p<.001\right]$. Although the main effect of coordinative working memory capacity did not reach the conventional level of significance $[F(1,40)=3.14$, $p=.08]$, the mean difference of the counting error index between low and high capacity participants (Table 3) suggests that the counting task may have been easier for the participants with high coordinative working memory capacity than for the low-capacity participants. There were no signs of an interaction between the coordinative demands of the counting task and the participants' individual coordinative capacity $[F(1,40)=0.62]$.

As in the previous experiments, the duration reproduction error correlated with the estimated number of stimulus lists the participants believed had been presented during the counting task $(r=.36, p<.05)$. In postexperimental interviews, all the participants reported that it was impossible for them to keep count of the stimulus lists while counting the targets.

\section{Discussion}

This experiment was designed to anchor the concept of coordinative demands within the context of individual differences in working memory capacity. The experimentally induced absorption of coordinative resources (by the counting task) yielded effects similar to those for naturally occurring individual differences in coordinative working memory capacity with regard to performance in both the counting task and the duration reproduction task. This finding is evidence for the validity of the coordinative demands construct. Moreover, it highlights the external validity of the present results, suggesting that associations between working memory and duration reproduction are not limited to artificial, experimentally controlled situations but can probably also be found in ecologically more valid settings.

\section{GENERAL DISCUSSION}

The attentional gate model holds that the remembered duration decreases as the attentional demands of the nontemporal task increase. This notion is based on a unitary concept of task difficulty and attentional demands. "When a relatively easy nontemporal task fills a duration, the amount of attentional resources left for opening the attentional gate is greater than when the task is more difficult" (Zakay \& Block, 1997, p. 15). Consequently, a variety of different experimental tasks has been used as easy or difficult nontemporal tasks. Zakay, Nitzan, and Glicksohn (1983), for example, found shorter duration estimates when participants were asked to read words (presumably an easy task) than when they were asked to give associations for low-frequency words (presumably a difficult task). Zakay et al. assumed that, irrespective of their qualitative differences, both tasks tax a common pool of attentional resources.

This unitary concept of attentional demands and resources was criticized by Brown (1997) as being unsuitable for explaining asymmetric interference effects: "A more sophisticated framework than the attentional allocation model is needed to account for these effects" (Brown, 1997, p. 1133). On the basis of his results for asymmetric dual-task interference effects, Brown hypothesized that the processing of temporal information depends primarily on the availability of central executive resources. Precisely these resources are assumed to be required to coordinate the temporal and the nontemporal tasks, meaning that any concurrent nontemporal task can be assumed to impair duration reproduction. However, nontemporal tasks may vary in the extent to which they tax central executive resources. Therefore, not all nontemporal tasks are necessarily affected by a concurrent timing task.

In the present experiments, an attempt was made to evaluate the unspecific (Zakay \& Block, 1997) and the specific (Brown, 1997) resource approaches, using time reproduction as a temporal task and multiple counting as a nontemporal task. The latter task allowed coordinative and noncoordinative (sequential) demands on the working memory system to be varied independently. Therefore, it was possible to test to what extent (1) increased costs of coordinating the temporal and the nontemporal tasks and (2) coordination-independent demands of the nontemporal task affected temporal information processing. The pattern of results corroborates the specific resource approach. All four experiments showed that, under increased coordinative demands on working memory, the remembered duration decreased when the difficulty of the nontemporal (counting) task increased. Moreover, increased sequential (noncoordinative) demands on working memory were shown to intensify the difficulty of the nontemporal task (Experiments 1 and 2). However, this manipulation did not affect the accuracy of the reproduced duration - a clear indication that not all kinds of attentional demands influence the remembered duration, but mainly those that increase the difficulty of dual-task coordination and, thus, tax the resources assumed to be required for constructing a reliable record of temporal cues. In terms of Baddeley's (1986) working memory model, a nontemporal task is expected to impair time reproduction performance the more this task requires the involvement of the central executive (see Brown, 1997).

Although the present results are confined to (prospective) time reproductions, it is interesting to note that they are in line with experiments on other processes in temporal cognition, such as time production rather than repro- 
duction. Fortin, Rousseau, Bourque, and Kirouac (1993), for example, found that a search task demanding a high level of attention interfered with time production performance only if the search task had to be processed in shortterm memory. More precisely, Fortin and Breton (1995) showed that demands on the processing component, rather than the storage component, of working memory affected time production. Both studies indicate that some kinds of demands on working memory affect time production, whereas others do not. These results also support the specific resource approach of temporal information processing. Thus, there seems to be converging evidence that models of working memory (e.g., Miyake \& Shah, 1999) can be used to specify under which conditions nontemporal tasks affect attention allocation processes and thereby influence the processing of temporal information. Working memory researchers have been extremely productive in creating particular tasks requiring activity of different working memory components (e.g., Oberauer et al., 2000). These tasks offer an excellent toolbox for analyzing the relations between nontemporal task demands and timing.

Whereas in many experiments in which attention allocation models have been studied stimulus intervals lasting milliseconds or seconds have been used, in the present experiments the stimulus intervals lasted several minutes. Because the nontemporal counting task used in the present experiments emphasizes demands on working memory across a number of stimulus lists, the stimulus interval is necessarily longer than that in experiments in which other concurrent tasks are used. The disadvantage is that the present results cannot directly be compared with experiments on the estimation of short intervals. On the other hand, the present results extend the scope of attention allocation models to the investigation of long intervals and confirm that the general prediction that increased cognitive demands impair duration reproduction also holds for these long intervals. However, the specific prediction that primarily coordinative demands also impair the reproduction of shorter intervals remains to be tested in future studies.

The present results were obtained with an experimental procedure that allows coordinative and noncoordinative demands on working memory to be manipulated within the same task. In previous studies, qualitatively different tasks have often been used to implement different levels of difficulty (or mental load) in the nontemporal task (e.g., Hicks \& Brundige, 1974; Kowal, 1981; Zakay et al., 1983). Since these tasks can be assumed to differ in several respects, it is hard to analyze what makes them more or less difficult and to what extent they tax the resources required for temporal information processing. The differences between the levels of coordinative and sequential demands in the counting task can be described in precise terms, however. Moreover, the demands of the counting task seem to correspond to naturally occurring individual differences in working memory resources, as is shown by the parallel results obtained by manipulating coordinative demands, on the one hand, and sampling participants with higher and lower coordinative working memory capacity, on the other.

To summarize, the present experiments provide additional evidence to show that temporal cognition involves working memory. As has been proposed by Brown (1997) and Fortin and Breton (1995), attention allocation models of time estimation, including the attentional gate model, may benefit from connections being forged to the flourishing field of working memory research. This may help to further specify the cognitive processes addressed by the metaphor of directing attention to time.

\section{REFERENCES}

AlLEN, J. A. (1980). Effects of increased noise levels by supersonic aircraft on annoyance levels and time estimations. Perceptual \& Motor Skills, 50, 563-569.

BADDELEY, A. [D.] (1986). Working memory. Oxford: Oxford University Press, Clarendon Press.

BADDELEY, A. D. (1992). Is working memory working? The fifteenth Bartlett lecture. Quarterly Journal of Experimental Psychology, 44A, $1-31$.

Baddeley, A. D. (2000). The episodic buffer: A new component of working memory? Trends in Cognitive Sciences, 4, 417-423.

BLock, R. A. (1990). Models of psychological time. In R. A. Block (Ed.), Cognitive models of psychological time (pp. 1-35). Hillsdale, NJ: Erlbaum.

Block, R. A., \& ZAKAY, D. (1996). Models of psychological time revisited. In H. Helfrich (Ed.), Time and mind (pp. 171-195). Seattle: Hogrefe \& Huber.

Brown, S. W. (1997). Attentional resources in timing: Interference effects in concurrent temporal and nontemporal working memory tasks. Perception \& Psychophysics, 59, 1118-1140.

Brown, S. W., \& West, A. N. (1990). Multiple timing and the allocation of attention. Acta Psychologica, 75, 103-121.

Decety, J., Jeannerod, M., \& Prablanc, C. (1989). The timing of mentally represented actions. Behavioural Brain Research, 34, 35 42.

Dutke, S., \& StöBer, J. (2001). Test anxiety, working memory, and cognitive performance: Supportive effects of sequential demands. Cognition \& Emotion, 15, 381-389.

Fortin, C., \& Breton, R. (1995). Temporal interval production and processing in working memory. Perception \& Psychophysics, 57, 203-215.

Fortin, C., Rousseau, R., Bourque, P., \& Kirouac, E. (1993). Time estimation and concurrent nontemporal processing: Specific interference from short-term-memory demands. Perception \& Psychophysics, 53, 536-548.

Fraisse, P. (1984). Perception and estimation of time. Annual Review of Psychology, 35, 1-36.

FREYD, J. J. (1992). Dynamic representations guiding adaptive behavior. In F. Macar, V. Pouthas, \& W. J. Friedman (Eds.), Time, action and cognition: Towards bridging the gap (pp. 309-323). Dordrecht: Kluwer.

Gibbon, J., Church, R. M., \& Meck, W. H. (1984). Scalar timing in memory. In J. Gibbon \& L. Allan (Eds.), Timing and time perception (Annals of the New York Academy of Sciences, Vol. 423, pp. 52-77). New York: New York Academy of Sciences.

GUAY, M., \& WILBERG, R. B. (1983). Immediate reproduction of temporal information under four cognitive strategies. Perceptual \& Motor Skills, 56, 375-381.

Hagendorf, H., \& SÁ, B. (1995). Coordination in visual working memory. Psychological Research, 58, 294-306.

Hawkes, G. R., \& Sherman, S. J. (1972). Vigilance effects for duration judgments with two levels of task demand. Perceptual \& Motor Skills, 34, 351-356.

Hemmes, N. S., Brown, B. L., \& Kladopoulos, C. N. (2004). Time perception with and without a concurrent nontemporal task. Perception \& Psychophysics, 66, 328-341. 
Hicks, R. E., \& BRUndige, R. M. (1974). Judgments of temporal duration while processing verbal and physiognomic stimuli. Acta Psychologica, 38, 447-453.

Hicks, R. E., Miller, G. W., Gaes, G., \& Bierman, K. (1977). Concurrent processing demands and the experience of time-in-passing. American Journal of Psychology, 90, 431-446.

Hicks, R. E., Miller, G. W., \& Kinsbourne, M. (1976). Prospective and retrospective judgments of time as a function of amount of information processed. American Journal of Psychology, 89, 719-730.

HÜLSER, C. (1924). Zeitauffassung und Zeitschätzung verschieden ausgefüllter Intervalle unter besonderer Berücksichtigung der Aufmerksamkeitsablenkung [Time perception and time estimation of differently filled intervals with respect to distracted attention]. Archiv für die gesamte Psychologie, 49, 363-378.

KowAL, K. H. (1981). Growth of apparent duration: Effect of melodic and non-melodic tonal variation. Perceptual \& Motor Skills, 52, 803 817.

Kyllonen, P. C., \& Christal, R. E. (1990). Reasoning ability is (little more than) working memory capacity?! Intelligence, 14, 389-433.

MaYr, U., \& KLIEGL, R. (1993). Sequential and coordinative complexity: Age-based processing limitations in figural transformations. Journal of Experimental Psychology: Learning, Memory, \& Cognition, 19, 1297-1320.

Michon, J. A. (1985). The compleat time experiencer. In J. A. Michon \& J. L. Jackson (Eds.), Time, mind, and behavior (pp. 20-52). Berlin: Springer.

Michon, J. A. (1990). Implicit and explicit representations of time. In R. A. Block (Ed.), Cognitive models of psychological time (pp. 3758). Hillsdale, NJ: Erlbaum.

Miyake, A., \& Shah, P. (Eds.) (1999). Models of working memory: Mechanisms of active maintenance and executive control. Cambridge: Cambridge University Press.

MunZert, J. (2002). Temporal accuracy of mentally simulated transport movements. Perceptual \& Motor Skills, 94, 307-318.

Norman, D. A., \& Shallice, T. (1986). Attention to action: Willed and automatic control of behavior. In R. J. Davidson, G. E. Schwartz, \& D. Shapiro (Eds.), Consciousness and self-regulation: Advances in research and theory (pp. 1-18). New York: Plenum.

Oberauer, K. (1993). Die Koordination kognitiver Operationen: Eine Studie über die Beziehung zwischen Intelligenz und "working memory" [Coordination of cognitive operations: A study on the relation of intelligence and working memory]. Zeitschrift für Psychologie, 201, 57-84.

Oberauer, K., Süß, H.-M., Schulze, R., Wilhelm, O., \& Wittmann, W. W. (2000). Working memory capacity: Facets of a cognitive ability construct. Personality \& Individual Differences, 29, 1017-1045.

Poynter, D. (1989). Judging the duration of time intervals: A process of remembering segments of experience. In I. Levin \& D. Zakay (Eds.), Time and human cognition: A life-span perspective (pp. 305-331). Amsterdam: North-Holland.
Roeckelein, J. E. (2000). The concept of time in psychology: A resource book and annotated bibliography. Westport, CT: Greenwood.

Süß, H.-M., Oberauer, K., Wittmann, W. W., Wilhelm, O., \& SCHULze, R. (2002). Working-memory capacity explains reasoning ability - and a little bit more. Intelligence, 30, 261-288.

Thomas, E. A. C., \& Weaver, W. B. (1975). Cognitive processing and time perception. Perception \& Psychophysics, 17, 363-367.

Verhaeghen, P., Kliegl, R., \& Mayr, U. (1997). Sequential and coordinative complexity in time-accuracy functions for mental arithmetic. Psychology \& Aging, 12, 555-564.

Wilkening, F., Levin, I., \& Druyan, S. (1987). Children's counting strategies for time quantification and integration. Developmental Psychology, 23, 823-831.

Woodrow, H. (1933). Individual differences in the reproduction of temporal intervals. American Journal of Psychology, 45, 271-281.

Woodrow, H. (1951). Time perception. In S. S. Stevens (Ed.), Handbook of experimental psychology (pp. 1224-1236). New York: Wiley

ZAKAY, D. (1989). Subjective time and attentional resource allocation: An integrated model of time estimation. In I. Levin \& D. Zakay (Eds.), Time and human cognition: A life-span perspective (pp. 365397). Amsterdam: North-Holland.

ZAKAY, D. (1993). Relative and absolute duration judgments under prospective and retrospective paradigms. Perception \& Psychophysics, $\mathbf{5 4}, 656-664$.

ZAKAY, D., \& BLock, R. A. (1997). Temporal cognition. Current Directions in Psychological Science, 6, 12-16.

ZaKay, D., NitZan, D., \& Glicksohn, J. (1983). The influence of task difficulty and external tempo on subjective time estimation. Perception \& Psychophysics, 34, 451-456.

\section{NOTES}

1. Recently, this model has been extended to include the so-called episodic buffer (Baddeley, 2000), which is not considered in the present context.

2 . In the counting task, four types of counting errors may occurnamely, (1) a no response when a target number appears for the third time, (2) a yes response when a target appears for the first or the second time only, (3) a yes response when the list does not contain a target, and (4) no response at all. Preliminary analyses failed to identify any effects of the type of counting error. Thus, all types of counting errors were collapsed in a single counting error index. This index reflects the total number of counting errors in relation to the number of lists in which a particular type of error could occur. Errors of Type 1 above could occur only in lists in which a target appeared for the third time, Type 2 errors only in lists in which a target appeared for the first or the second time, and Type 3 errors only in lists containing no targets.

(Manuscript received October 2, 2003; revision accepted for publication February 23, 2005.) 